\title{
Universal theory of efficiency fluctuations
}

\author{
Gatien Verley, ${ }^{1}$ Tim Willaert, ${ }^{2}$ Christian Van den Broeck, ${ }^{2}$ and Massimiliano Esposito ${ }^{1}$ \\ ${ }^{1}$ Complex Systems and Statistical Mechanics, University of Luxembourg, L-1511 Luxembourg, G.D. Luxembourg \\ ${ }^{2}$ Hasselt University, B-3590 Diepenbeek, Belgium \\ (Received 16 September 2014; published 21 November 2014)
}

\begin{abstract}
Using the fluctuation theorem supplemented with geometric arguments, we derive universal features of the (long-time) efficiency fluctuations for thermal and isothermal machines operating under steady or periodic driving, close or far from equilibrium. In particular, the probabilities for observing the reversible efficiency and the least likely efficiency are identical to those of the same machine working under the time-reversed driving. For time-symmetric drivings, this reversible and the least probable efficiency coincide.
\end{abstract}

DOI: 10.1103/PhysRevE.90.052145

PACS number(s): 05.70.Ln, 05.40.-a, 84.60.-h

\section{INTRODUCTION}

A thermodynamic machine is designed to operate for a given purpose such as producing mechanical work or cooling. This statement is correct "on average," meaning that fluctuations might occasionally prevent the machine from operating in the same way as the average behavior. While fluctuations are negligible when considering macroscopic machines, they become highly relevant at small scales when considering molecular machines or nanodevices. The macroscopic efficiency $\bar{\eta}$ used to characterize the performance of a machine "on average" is defined as the ratio between an average output and an average input contribution to the macroscopic entropy production (EP) of the machine. A central result in macroscopic thermodynamics is that the second law imposes an upper bound to this macroscopic efficiency, which is only reached when the machine operates reversibly. Stochastic thermodynamics has taught us that entropy production, and thus its output and input contributions, can be defined at the level of single stochastic trajectories [1-6]. In this paper we study the statistical properties of stochastic efficiencies defined at the trajectory level as ratios between such output and input. These efficiencies may be negative or higher than the reversible efficiency, corresponding to large fluctuations along which the machine does not operate in the same mode as the average behavior. However, the fluctuation theorem [7-10] imposes constraints on their probability distribution, more precisely on their large deviation function (LDF). Remarkably, the shape of the efficiency LDF is quite generic and displays universal features: The long-time probabilities for observing the reversible efficiency and the least likely efficiency are identical to those of the same machine working under the time-reversed driving. In the special case of machines operating at steady states or under time-symmetric drivings, the reversible efficiency coincides with the least probable and the main result predicted in Ref. [11] is recovered. In this paper, besides proving these general results, we also provide an efficient method to calculate the efficiency LDF and extensively illustrate our results on an exactly solvable driven two-level system. We note that Ref. [12], which appeared while finalizing this paper, provides further numerical evidence (see Fig. 3) that the forward and time-reversed efficiency distributions cross at the reversible efficiency on a model similar to ours.

After framing the basic issue in Sec. II, the general theory and the main results are derived in Sec. III. Model-system illustrations of these are provided in Sec. IV, and conclusions are drawn in Sec. V.

\section{THERMODYNAMICS OF NANOMACHINES}

To study efficiency fluctuations of small-scale machines, we consider the following generic setup. A small-scale machine is subjected to two thermodynamic forces $A_{1}$ and $A_{2}$, inducing over a certain time $t$, the conjugated time-integrated currents $\mathcal{J}_{1}$ and $\mathcal{J}_{2}$, being positive when flowing toward the machine. While thermodynamic forces are expressed in terms of nonfluctuating properties of the macroscopic reservoirs, the currents, and hence also the efficiency, will typically fluctuate. These currents induce a fluctuating EP $\Sigma=\Sigma_{1}+\Sigma_{2}+\Delta S$, where $\Sigma_{1}=A_{1} \mathcal{J}_{1}, \Sigma_{2}=A_{2} \mathcal{J}_{2}$, and $\Delta S$ is the stochastic entropy change of the machine itself. Integrated currents and EP are time-extensive (i.e., over long times they typically grow and their average increases linearly with time). We consider small machines with finite state space, meaning that the entropy changes $\Delta S$ become negligible in the long time limit (they can be shown to vanish in a large deviation sense). Their EP over long times thus reads

$$
\Sigma \sim \mathcal{J}_{1} A_{1}+\mathcal{J}_{2} A_{2} .
$$

Machines operate either steadily or cyclically with period $\tau$. In this latter case, time is expressed in terms of the number of periods $n$ as $t=n \tau$. We denote the time-intensive variables by a lower case, e.g., $j_{1}=\mathcal{J}_{1} / t$ or $j_{2}=\mathcal{J}_{2} / t, \sigma=\Sigma / t, \sigma_{1}=$ $\Sigma_{1} / t$ or $\sigma_{2}=\Sigma_{2} / t$, and ensemble averages by brackets $\langle.$.$\rangle .$

A device operating as a machine (in average) uses a fueling process (the input) flowing in the spontaneous direction of its corresponding forces $\left\langle\sigma_{1}\right\rangle>0$ (e.g., a heat flowing down a temperature gradient or particle flowing down a chemical potential gradient) in order to power a second process (the output) flowing against the spontaneous direction of its corresponding forces $\left\langle\sigma_{2}\right\rangle<0$ (e.g., a particles flowing up a chemical potential gradient or a coordinate moving against the direction of a mechanical force). Since the second law imposes $\langle\sigma\rangle \geqslant 0$, the ("type II" [13]) macroscopic efficiency of the machine defined as

$$
\bar{\eta}=-\frac{\left\langle\sigma_{2}\right\rangle}{\left\langle\sigma_{1}\right\rangle}=-\frac{A_{2}\left\langle j_{2}\right\rangle}{A_{1}\left\langle j_{1}\right\rangle} \leqslant 1
$$

is always bounded upwards by the reversible (or Carnot) efficiency $\bar{\eta}_{\text {rev }}=1$ occurring when $\langle\sigma\rangle \rightarrow 0$. We note that 
TABLE I. Currents and affinities in the EP decomposition of Eq. (1) with $\Sigma_{1}=\mathcal{J}_{1} A_{1}$ and $\Sigma_{2}=\mathcal{J}_{2} A_{2}$ for thermal machines operating, respectively, as heat engine, heat pump, and refrigerator. The input and output currents $\left\langle\mathcal{J}_{1}\right\rangle$ and $-\left\langle\mathcal{J}_{2}\right\rangle$ are always positive on average. $\eta_{C}=1-T_{c} / T_{h}$ is the Carnot efficiency. Type I efficiency is defined as $-\left\langle\mathcal{J}_{2}\right\rangle /\left\langle\mathcal{J}_{1}\right\rangle$, which is always smaller or equal to $A_{1} / A_{2}$. Type II efficiency, cf. Eq. (2), is equal to type I efficiency divided by $A_{1} / A_{2}$, hence always smaller or equal to 1 .

\begin{tabular}{cccc}
\hline \hline & Heat engine & Heat pump & Refrigerator \\
\hline $\mathcal{J}_{1}$ & $Q_{h}$ & $W$ & $W$ \\
$A_{1}$ & $\eta_{C} / T_{c}$ & $1 / T_{c}$ & $1 / T_{h}$ \\
$\mathcal{J}_{2}$ & $W$ & $Q_{h}$ & $-Q_{c}$ \\
$A_{2}$ & $1 / T_{c}$ & $\eta_{C} / T_{c}$ & $\eta_{C} / T_{c}$ \\
\hline \hline
\end{tabular}

traditional efficiencies ("type I" [13]) can be trivially recovered from these efficiencies; see Table I for the case of thermal machines.

In the same spirit, we introduce the following time-intensive dimensionless quantity, called the stochastic efficiency:

$$
\eta=-\frac{\sigma_{2}}{\sigma_{1}}=-\frac{A_{2} j_{2}}{A_{1} j_{1}} .
$$

As we will see below, its most probable value converges in the long time limit to its macroscopic value $\eta \rightarrow \bar{\eta}$. To investigate the approach to this limit, we focus on the convergence of the intensive EPs $\sigma_{1}$ and $\sigma_{2}$ to their most probable value, which also coincides with their average. Large deviation theory describes the exponential decay of the probability $P_{t}\left(\sigma_{1}, \sigma_{2}\right)$ for observing nontypical (i.e., different from their infinite-time average) EPs:

$$
P_{t}\left(\sigma_{1}, \sigma_{2}\right) \asymp \exp \left\{-t I\left(\Sigma_{1}, \Sigma_{2}\right)\right\}
$$

The rate $I\left(\sigma_{1}, \sigma_{2}\right)$ is the nonnegative and convex LDF, which reaches its minimum value at the point $\sigma_{1}=\left\langle\sigma_{1}\right\rangle$ and $\sigma_{2}=\left\langle\sigma_{2}\right\rangle$, which carries the entire probability weight $I\left(\left\langle\sigma_{1}\right\rangle,\left\langle\sigma_{2}\right\rangle\right)=0$.

A central result in stochastic thermodynamics known as the fluctuation theorem states that the probability to observe a positive EP in a driven machine is exponentially more likely than that of observing its negative counterpart when the machine is subjected to the time-reversed driving. This result implies that any decomposition of the EP into subparts that are antisymmetric under time reversal (as is the case for $\left.\Sigma=\Sigma_{1}+\Sigma_{2}\right)$ inherits the same symmetry property [14,15]. Expressed in the framework of large deviation theory, the fluctuation theorem takes the following form (we set $k_{B}=1$ ):

$$
I\left(\sigma_{1}, \sigma_{2}\right)-\hat{I}\left(-\sigma_{1},-\sigma_{2}\right)=-\sigma_{1}-\sigma_{2},
$$

where $\hat{P}_{t}\left(\sigma_{1}, \sigma_{2}\right) \asymp \exp \left\{-t \hat{I}\left(\sigma_{1}, \sigma_{2}\right)\right\}$ is the probability of the EPs $\sigma_{1}$ and $\sigma_{2}$ for the machine working with the time-reversed driving cycle. For symmetric driving cycles or for steady machines, we obviously have that $I\left(\sigma_{1}, \sigma_{2}\right)=\hat{I}\left(\sigma_{1}, \sigma_{2}\right)$. In the next sections, we study the implications of this result for efficiency fluctuations.

\section{EFFICIENCY FLUCTUATIONS}

\section{A. Large deviation function of efficiency}

We start by deriving the efficiency LDF from the EPs LDF $I\left(\sigma_{1}, \sigma_{2}\right)$. The probability to observe an efficiency $\eta$ is given by

$$
\begin{aligned}
P_{t}(\eta) & =\int d \sigma_{1} d \sigma_{2} P_{t}\left(\sigma_{1}, \sigma_{2}\right) \delta\left(\eta+\frac{\sigma_{2}}{\sigma_{1}}\right) \\
& =\int d \sigma_{1} P_{t}\left(\sigma_{1},-\eta \sigma_{1}\right)\left|\sigma_{1}\right| .
\end{aligned}
$$

Inserting Eqs. (4) into (6) and using the Laplace approximation to compute the integral exactly in the long time limit, we find that

$$
P_{t}(\eta) \asymp \exp \{-t J(\eta)\}
$$

where

$$
J(\eta)=\min _{\sigma_{1}} I\left(\sigma_{1},-\eta \sigma_{1}\right) .
$$

This result implies that $J(\eta) \leqslant I(0,0), \forall \eta$, an important property to be used in the next section. Furthermore, it follows that the efficiency LDF $J(\eta)$ vanishes at the macroscopic efficiency $\bar{\eta}$, thus corresponding to the most probable efficiency:

$$
J(\bar{\eta})=\min _{\sigma_{1}} I\left(\sigma_{1}, \sigma_{1} \frac{\left\langle\sigma_{2}\right\rangle}{\left\langle\sigma_{1}\right\rangle}\right)=0 .
$$

The minimum value zero is reached for $\sigma_{1}=\left\langle\sigma_{1}\right\rangle$ because the LDF for EPs is convex and vanishes at the average EPs.

\section{B. Geometric interpretation}

We now analyze the contour lines of the convex LDF $I\left(\sigma_{1}, \sigma_{2}\right)$ in the input and output $\left(\sigma_{1},-\sigma_{2}\right)$ plane represented in Fig. 1. They form closed convex lines encircling the point $\mathrm{C}=\left(\left\langle\sigma_{1}\right\rangle,-\left\langle\sigma_{2}\right\rangle\right)$, where $I$ reaches its minimal value $I=0$; see Fig. 1. This point corresponds to the most probable efficiency $\eta=\bar{\eta}$ and must lie in the upper-right corner of the $\left(\sigma_{1},-\sigma_{2}\right)$ plane for the device to operate as a machine. A given value of the stochastic efficiency corresponds to a straight line with slope $\eta$ and crossing the origin: $-\sigma_{2}=\eta \sigma_{1}$. The corresponding value of the LDF $J(\eta)$ is, according to Eq. (8), found as the minimum of $I\left(\sigma_{1}, \sigma_{2}\right)$ along this line. This minimum is reached for the contour line closest to the most probable point $\mathrm{C}$, namely the contour tangent to the line of slope $\eta$. A given contour line has two tangent lines crossing the origin and corresponding to two different efficiencies with the same value of the LDF. For instance, in Fig. 1 the black solid contour line has two tangent lines, one in $\mathrm{B}$ and one in $\mathrm{D}$, corresponding to the efficiencies $\eta_{B}$ and $\eta_{D}$ and to the same value of the LDF $J\left(\eta_{B}\right)=J\left(\eta_{D}\right)$ as shown in Fig. 2.

From the above geometric analysis of Eq. (8) illustrated in Fig. 1, we can deduce the overall typical shape of the efficiency LDF $J(\eta)$ represented in Fig. 2. Starting from the point $\mathrm{C}$ with efficiency $\eta=\bar{\eta}$ and decreasing the slope $\eta, J(\eta)$ increases until the contour line touches the vertical axis in point A, with the corresponding efficiency $\eta=-\infty$. Similarly, increasing the slope $\eta$ from $\mathrm{C}$ upward, the LDF $J(\eta)$ increases until the contour line crosses the origin corresponding to $I(0,0)=J\left(\eta^{*}\right)$, where $\eta^{*}$ is the contour slope at the origin. 


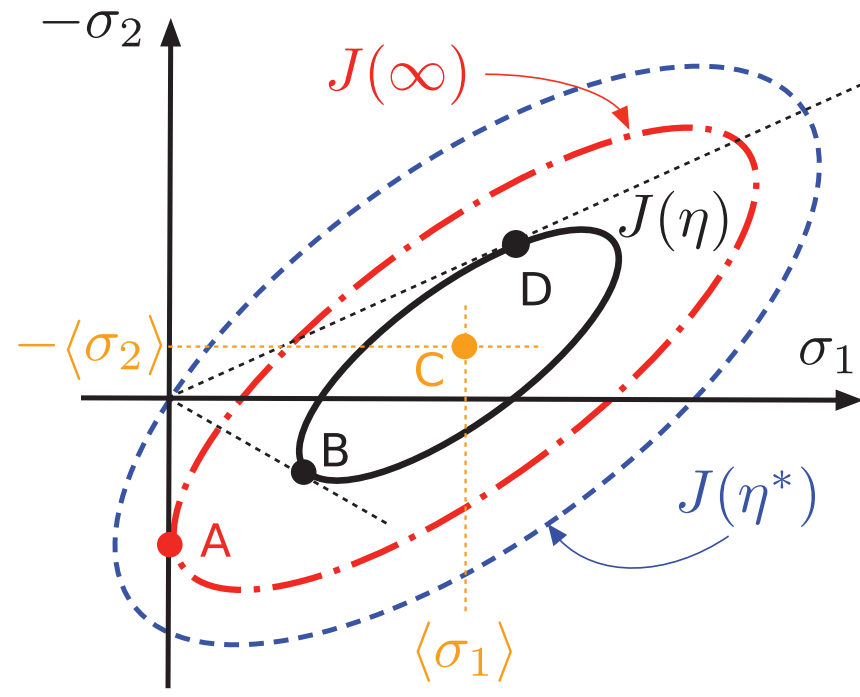

FIG. 1. (Color online) Typical contour lines of the $\operatorname{LDF} I\left(\sigma_{1}, \sigma_{2}\right)$. The point $\mathrm{C}$ corresponds to the most probable value $I\left(\left\langle\sigma_{1}\right\rangle,\left\langle\sigma_{2}\right\rangle\right)=0$. A straight line through the origin with slope $\eta_{D}$ touches the contour line, whose $I$ value equals $J\left(\eta_{D}\right)$ (idem for point B sharing the same $J$ value). The maximum of $J(\eta)$ corresponds to $I$ value of the contour crossing the origin $J\left(\eta^{*}\right)=I(0,0)$ (blue long dashed line), while $J(\infty)$ to that of the contour touching the $\sigma_{2}$ axis in A (red dash-dotted line).

This efficiency corresponds, as shown above, to the maximum value of $J(\eta)$, hence $\eta^{*}$ is the least-probable efficiency in the sense of large deviations. For $\eta \geqslant \eta^{*}$, the intersection between the contour and the efficiency line moves from the upper-right corner to the lower-left corner of the plane, and the LDF decreases until its limiting value is reached for $\eta=+\infty$. Positive and negative infinite efficiencies share the same contour line touching the vertical axis in A, with the same limiting $J(\infty)$ value.

\section{Least likely and reversible efficiency}

We have shown that the least probable efficiency is given by the slope in zero of the contour line crossing the origin.

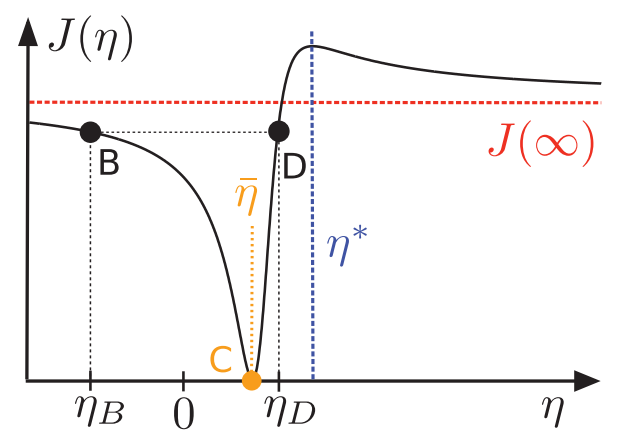

FIG. 2. (Color online) Typical shape of the efficiency LDF $J(\eta)$. For steady-state machines or machines with time-symmetric driving cycles, the shape is the same and the maximum is at the reversible efficiency $\eta^{*}=\bar{\eta}_{\text {rev }}=1$. The horizontal asymptote corresponds to point A of Fig. 1.
Along a contour line the total differential of $I$ has to vanish:

$$
d I=\frac{\partial I}{\partial \sigma_{1}} d \sigma_{1}+\frac{\partial I}{\partial \sigma_{2}} d \sigma_{2}=0 .
$$

Evaluating this equation at the origin one gets

$$
\eta^{*}=-\frac{d \sigma_{2}}{d \sigma_{1}}=\frac{\partial I}{\partial \sigma_{1}}\left(\frac{\partial I}{\partial \sigma_{2}}\right)^{-1},
$$

and similarly for the machine subjected to the time-reversed driving cycle

$$
\hat{\eta}^{*}=-\frac{d \sigma_{2}}{d \sigma_{1}}=\frac{\partial \hat{I}}{\partial \sigma_{1}}\left(\frac{\partial \hat{I}}{\partial \sigma_{2}}\right)^{-1},
$$

where $\eta^{*}$ and $\hat{\eta}^{*}$ are defined by $J\left(\eta^{*}\right)=I(0,0)$ and $\hat{J}\left(\hat{\eta}^{*}\right)=$ $\hat{I}(0,0)$. Taking the partial derivative with respect to $\sigma_{1}$ and $\sigma_{2}$ of the fluctuation theorem Eq. (5) and evaluating it at vanishing EPs leads to the following two equations:

$$
\frac{\partial I}{\partial \sigma_{1}}+\frac{\partial \hat{I}}{\partial \sigma_{1}}=-1, \quad \frac{\partial I}{\partial \sigma_{2}}+\frac{\partial \hat{I}}{\partial \sigma_{2}}=-1 .
$$

Therefore, the least probable efficiency of the machine subjected to the time-reversed driving cycle is related to the EPs LDF of the original machine by

$$
\hat{\eta}^{*}=\left(1+\frac{\partial I}{\partial \sigma_{1}}\right)\left(1+\frac{\partial I}{\partial \sigma_{2}}\right)^{-1} .
$$

For machines operating at steady state or subjected to time-symmetric driving cycles, $I\left(\sigma_{1}, \sigma_{2}\right)=\hat{I}\left(\sigma_{1}, \sigma_{2}\right)$ and from Eq. (13), one recovers the result first derived in Ref. [11] stating that the least probable efficiency is the reversible efficiency: $\eta^{*}=\hat{\eta}^{*}=\bar{\eta}_{\mathrm{rev}}=1$. However, if the machine works with nontime-symmetric cyclic driving, the reversible efficiency is not the least probable any more but remains a special point of the LDF. Indeed, if we evaluate Eq. (5) in $\sigma_{2}=-\bar{\eta}_{\mathrm{rev}} \sigma_{1}=-\sigma_{1}$, we find that

$$
I\left(\sigma_{1},-\bar{\eta}_{\mathrm{rev}} \sigma_{1}\right)=\hat{I}\left(-\sigma_{1}, \bar{\eta}_{\mathrm{rev}} \sigma_{1}\right)
$$

which after minimization over $\sigma_{1}$ implies that

$$
J\left(\bar{\eta}_{\text {rev }}\right)=\hat{J}\left(\bar{\eta}_{\text {rev }}\right) .
$$

Hence, the efficiency LDF takes the same value at the reversible efficiency for both the forward and the reversed machine. The two LDFs will thus cross at this point. Furthermore, using the fluctuation theorem Eq. (5), we find that $I(0,0)=\hat{I}(0,0)$. This implies that the maximum of the forward and reversed efficiency LDF have the same value:

$$
J\left(\eta^{*}\right)=\hat{J}\left(\hat{\eta}^{*}\right)
$$

These two central results, Eqs. (16) and (17), show that the fluctuation theorem imposes strong universal constraints on efficiency fluctuations as illustrated in Fig. 3.

\section{Efficiency LDF from EPs cumulant generating function}

We now propose a convenient way to calculate the efficiency LDF directly using the EPs cumulant generating function (CGF):

$$
\phi\left(\gamma_{1}, \gamma_{2}\right)=\lim _{t \rightarrow \infty} \frac{1}{t} \ln \left\langle e^{\gamma_{1} \Sigma_{1}+\gamma_{2} \Sigma_{2}}\right\rangle .
$$




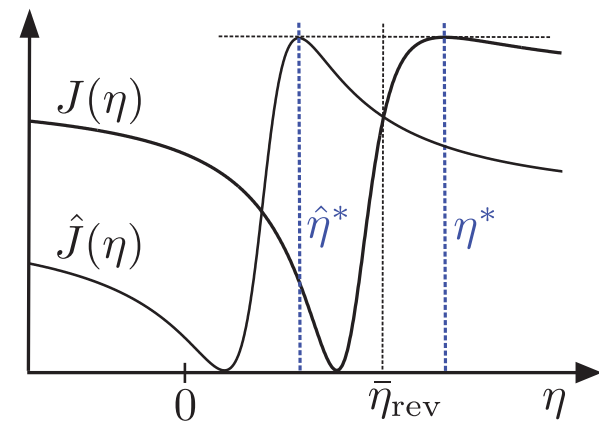

FIG. 3. (Color online) Typical shape of the efficiency LDF $J(\eta)$ and $\hat{J}(\eta)$. The two curves cross at $\eta=\bar{\eta}_{\text {rev }}=1$ and take the same maximum value at their least likely efficiency $\eta^{*}$ and $\hat{\eta}^{*}$.

This latter is typically obtained analytically or numerically from the dominant eigenvalue of a dressed stochastic generator [16-18]. The CGF and LDF for EPs are known to be related by a Legendre transform [19]:

$$
\begin{gathered}
\phi\left(\gamma_{1}, \gamma_{2}\right)=\max _{\sigma_{1}, \sigma_{2}}\left\{\gamma_{1} \sigma_{1}+\gamma_{2} \sigma_{2}-I\left(\sigma_{1}, \sigma_{2}\right)\right\}, \\
I\left(\sigma_{1}, \sigma_{2}\right)=\max _{\gamma_{1}, \gamma_{2}}\left\{\gamma_{1} \sigma_{1}+\gamma_{2} \sigma_{2}-\phi\left(\gamma_{1}, \gamma_{2}\right)\right\} .
\end{gathered}
$$

Therefore, the minimization of Eq. (8) can be directly performed on

$$
I\left(\sigma_{1},-\eta \sigma_{1}\right)=\max _{\gamma_{1}, \gamma_{2}}\left\{\left(\gamma_{1}-\gamma_{2} \eta\right) \sigma_{1}-\phi\left(\gamma_{1}, \gamma_{2}\right)\right\} .
$$

Using the change of variable $\gamma=\gamma_{1}-\gamma_{2} \eta$ and the efficiency LDF definition, we get

$$
J(\eta)=\min _{\sigma_{1}}\left(\max _{\gamma}\left\{\gamma \sigma_{1}+\max _{\gamma_{2}}\left[-\phi\left(\gamma+\gamma_{2} \eta, \gamma_{2}\right)\right]\right\}\right) .
$$

Defining the function

$$
f_{\eta}(\gamma)=-\max _{\gamma_{2}}\left[-\phi\left(\gamma+\gamma_{2} \eta, \gamma_{2}\right)\right]=\min _{\gamma_{2}} \phi\left(\gamma+\gamma_{2} \eta, \gamma_{2}\right),
$$

whose Legendre transform is such that

$$
\begin{aligned}
& \mathcal{F}_{\eta}\left(\sigma_{1}\right)=\max _{\gamma}\left\{\gamma \sigma_{1}-f_{\eta}(\gamma)\right\}, \\
& f_{\eta}(\gamma)=\max _{\sigma_{1}}\left\{\gamma \sigma_{1}-\mathcal{F}_{\eta}(\gamma)\right\},
\end{aligned}
$$

the efficiency LDF can be written as:

$$
\begin{aligned}
J(\eta) & =\min _{\sigma_{1}}\left\{\max _{\gamma}\left[\gamma \sigma_{1}-f_{\eta}(\gamma)\right]\right\}, \\
& =\min _{\sigma_{1}} \mathcal{F}_{\eta}\left(\sigma_{1}\right), \\
& =-\max _{\sigma_{1}}\left\{-\mathcal{F}_{\eta}\left(\sigma_{1}\right)\right\}, \\
& =-f_{\eta}(0) .
\end{aligned}
$$

Using Eq. (22), we finally conclude that

$$
J(\eta)=-\min _{\gamma_{2}} \phi\left(\gamma_{2} \eta, \gamma_{2}\right) .
$$

This result is of significant practical importance because it shows that the efficiency LDF can be obtained using a simple minimization procedure from the EPs CGF, which can be calculated using well-known conventional techniques.

\section{E. Efficiency fluctuations close to equilibrium}

Close to equilibrium, the CGF of EPs is generically a quadratic function

$$
\phi\left(\gamma_{1}, \gamma_{2}\right)=\frac{1}{2} \sum_{i, k=1,2} C_{i k} \gamma_{i} \gamma_{k}+\sum_{k=1,2} \gamma_{k}\left\langle\sigma_{k}\right\rangle,
$$

with $C_{i k}$ the asymptotic value of the covariance matrix elements $C_{i k}(t)=\left(\left\langle\Sigma_{i}(t) \Sigma_{k}(t)\right\rangle-\left\langle\Sigma_{i}(t)\right\rangle\left\langle\Sigma_{k}(t)\right\rangle\right) / t$. The position of the minimum $\gamma_{2}^{*}$ in Eq. (26) is solution of $d \phi\left(\gamma_{2} \eta, \gamma_{2}\right) / d \gamma_{2}=0$ and reads

$$
\gamma_{2}^{*}=-\frac{\eta\left\langle\sigma_{1}\right\rangle+\left\langle\sigma_{2}\right\rangle}{\eta^{2} C_{11}+2 \eta C_{12}+C_{22}} .
$$

It follows from Eqs. (26), (27), and (28) that the efficiency LDF close to equilibrium is $J(\eta)=-\phi\left(\gamma_{2}^{*} \eta, \gamma_{2}^{*}\right)$, namely,

$$
J(\eta)=\frac{1}{2} \frac{\left(\eta\left\langle\sigma_{1}\right\rangle+\left\langle\sigma_{2}\right\rangle\right)^{2}}{\eta^{2} C_{11}+2 \eta C_{12}+C_{22}} .
$$

From linear response theory, currents are a linear combination of the affinities, $\left\langle j_{i}\right\rangle=\sum_{k=1,2} L_{i k} A_{k}$, with the Onsager coefficient defined by

$$
L_{i k}=\lim _{t \rightarrow \infty} \frac{1}{2 t}\left\langle\left[\mathcal{J}_{i}(t)-\left\langle\mathcal{J}_{i}\right\rangle_{\text {eq }}\right]\left[\mathcal{J}_{k}(t)-\left\langle\mathcal{J}_{k}\right\rangle_{\text {eq }}\right]\right\rangle_{\text {eq }} .
$$

They are related to the covariance matrix of EPs by $\lim _{t \rightarrow \infty} C_{i k}(t) / 2=A_{i} L_{i k} A_{k}$. Then, the average EPs are related to the asymptotic covariance matrix by $\left\langle\sigma_{i}\right\rangle=$ $\sum_{k=1,2} C_{i k} / 2$ so that Eq. (29) can be rewritten as

$$
J(\eta)=\frac{1}{8} \frac{\left[\eta C_{11}+(1+\eta) C_{12}+C_{22}\right]^{2}}{\eta^{2} C_{11}+2 \eta C_{12}+C_{22}} .
$$

This relation also results from combining the quadratic CGF of Eq. (27) with the fluctuation theorem

$$
\phi\left(\gamma_{1}, \gamma_{2}\right)=\hat{\phi}\left(-1-\gamma_{1},-1-\gamma_{2}\right),
$$

which is directly connected to Eq. (5). We have introduced $\hat{\phi}\left(\gamma_{1}, \gamma_{2}\right)$ the CGF for the machine working with the timereversed driving cycles. From the fact that Eq. (32) must hold for any couple $\left(\gamma_{1}, \gamma_{2}\right)$, we identify each monomial appearing in the quadratic forms of Eq. (32). Using these constraints and the exchange symmetry $1 \leftrightarrow 2$, we conclude that, close to equilibrium, the EPs CGF for both the drivings (direct and reverse) follow the same statistics $\phi\left(\gamma_{1}, \gamma_{2}\right)=\hat{\phi}\left(\gamma_{1}, \gamma_{2}\right)$. Therefore, we can generalize the result of Ref. [11] stating that the efficiency LDF Eq. (31) applies to both symmetric and nonsymmetric drivings. As a byproduct, we recover that $\left\langle\sigma_{i}\right\rangle=\sum_{k=1,2} C_{i k} / 2$ and Eq. (31).

\section{TWO-STATE CYCLIC MACHINE}

To illustrate the results of the previous section, we consider a system made of two states $\theta= \pm 1$ coupled to a cold and a hot heat reservoir $v=c, h$ at temperatures $T_{v}=1 / \beta_{v}$. The system energies $E(t)=-h(t) \theta(t)$ are modulated by an external piecewise constant driving $h(t)$ of period $\tau$, where $\theta(t)$ denotes the system state at time $t$. The energy changes in the system due to changes in the driving $h(t)$ (occurring at fixed $\theta$ ) constitute work. The energy changes between system states (occurring at a fixed driving value $h$ ) induced by either 


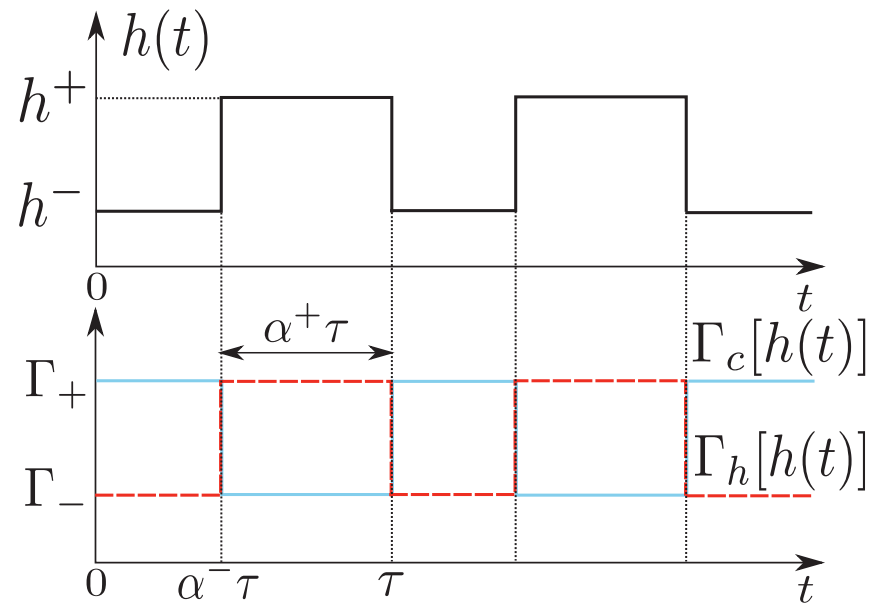

FIG. 4. (Color online) (Top) External driving $h(t)$ following a piecewise constant protocol of period $\tau$. The driving takes the value $h^{ \pm}=h_{0} \pm a$ during $\alpha^{ \pm} \tau$, with $\alpha^{-}+\alpha^{+}=1$. (Bottom) Time evolution of $\Gamma_{v}(t)$ indicating the coupling with cold reservoir $v=c$ for the blue solid line and with the hot reservoir $v=h$ for the red dashed line. At driving $h^{ \pm}$, the hot reservoir coupling is $\Gamma_{ \pm}$and the cold reservoir coupling is $\Gamma_{\mp}$.

reservoirs and occurring at random times constitute heat (work and heat are by convention positive when they increase the system energy). The Markovian rates describing these latter transitions from $\theta$ to $-\theta$ due to reservoir $v$ are of the form

$$
k_{\nu}[h(t), \theta]=\omega_{\nu}[h(t)] e^{-\beta_{v} h(t) \theta},
$$

and thus satisfy local detailed balance. We consider Fermi rates $\omega_{\nu}[h(t)]=\Gamma_{\nu}[h(t)] /\left[2 \cosh \beta_{\nu} h(t)\right]$, but Arrhenius rates $\omega_{\nu}[h(t)]=\Gamma_{\nu}[h(t)] /\left|2 \sinh \beta_{\nu} h(t)\right|$ or Bose rates $\omega_{\nu}[h(t)]=$ $\Gamma_{\nu}[h(t)] /\left|2 \sinh \beta_{\nu} h(t)\right|$ may be considered as well. In order for the system to operate as a thermal machine, the coupling constants $\Gamma_{\nu}[h(t)]$ between the system and the reservoir $v$ have to depend on the driving value. The heat per period received from the hot (respectively, cold) reservoir is denoted $q_{h}$ (respectively, $q_{c}$ ). The work per period performed by the driving on the system is denoted $w$. Table I describes the different possible operating regimes of our thermal machine and explains how to relate work and heat to the EPs and efficiency of the general formulation of Sec. II.

We first consider the piecewise constant driving depicted in Fig. 4. This driving is symmetric under time-reversal (up to a time shift negligible in the long time limit) and the single reservoir version of this model was studied analytically in Refs. [20,21]. The work and heat CGF can also be calculated analytically for our machine as described in the Appendix.

Depending on the choice of the various parameters, this machine operates in the different modes illustrated in Fig. 5. Note that refrigerators and heat pumps only differ by the way efficiency is defined: using either the heat from the cold reservoir or from the hot reservoir for output process. We also see that the heat pump and refrigerator region is separated from the heat engine region by two different dud engine regions. The red region corresponds to a heater using work to heat the hot and the cold reservoirs. The light beige region is also a dud engine that uses work to enhance the heat flow in its

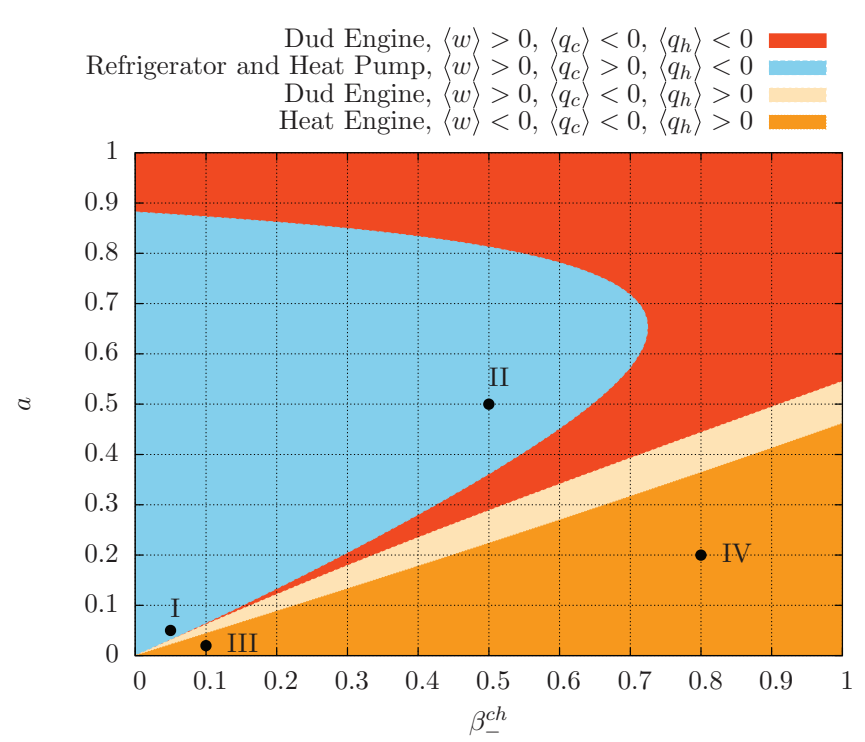

FIG. 5. (Color online) Diagram representing the various operating modes of our thermal machine subjected to the driving cycle depicted in Fig. 4, as a function of the inverse temperature difference $\beta_{-}^{c h}=\beta_{c}-\beta_{h}$ and the amplitude $a$ of the driving $\left(h^{ \pm}=h_{0} \pm a\right)$. We used: sum of inverse temperatures $\beta_{+}^{c h}=\beta_{c}+\beta_{h}=2$, period $\tau=1$, cyclic ratio $\alpha^{-}=0.3$, bare field $h_{0}=1$, and coupling constants $\Gamma_{-}=0.25$ and $\Gamma_{+}=4$. These parameters are also used for all figures using this model (excepted Fig. 10). Black dots correspond to the values of $a$ and $\beta_{-}^{c h}$ used in Figs. 6, 7, and 8. I: $\beta_{-}^{c h}=0.05, a=0.05$; II: $\beta_{-}^{c h}=0.5, a=0.5$; III: $\beta_{-}^{c h}=0.1, a=0.02$; IV: $\beta_{-}^{c h}=0.8$, $a=0.2$.

spontaneous direction. The two black dots in the blue as well as in the orange region correspond to the close-to-equilibrium (dots I and III) and far-from-equilibrium (dots II and IV) parameter values.

Using Table II and the exact CGF derived for work and heat in the Appendix, the efficiency LDF $J(\eta)$ can be directly obtained by a numerical minimization as suggested by Eq. (26). Alternatively, one could also directly minimize the LDF $I\left(\sigma_{1}, \sigma_{2}\right)$ computed via a two-dimensional Legendre transform of the joint CGF.

In Fig. 6, the $\operatorname{LDF} I\left(\sigma_{1}, \sigma_{2}\right)$ is displayed for the three operating modes of the machine, both close and far from equilibrium. Since the driving depicted in Fig. 4 is symmetric under time-reversal, i.e., $I\left(\sigma_{1}, \sigma_{2}\right)=\hat{I}\left(\sigma_{1}, \sigma_{2}\right)$, we verify Eq. (5) and obtain that the least probable efficiency coincides with the reversible efficiency (given by the slope of the contour lines crossing the origin). The fact that the contour lines become elliptical close to equilibrium entails Gaussian statistics for the EPs, $\sigma_{1}$ and $\sigma_{2}$.

In Fig. 7 (respectively, Fig. 8), we plot $J(\eta)$ for the three operating modes of the machine operating close to equilibrium (respectively, far from equilibrium) and corresponding to the black dots in Fig. 5. We verify that the reversible efficiency corresponds to a maximum of the LDF as it should for timesymmetric drivings. Figure 7 also confirms the validity of our close-to-equilibrium theory presented in Sec. III E. We observe that the curves for the refrigerator and the heat pump are very similar in the close-to-equilibrium limit. The plateau value for large efficiencies and the value of the efficiency LDF 

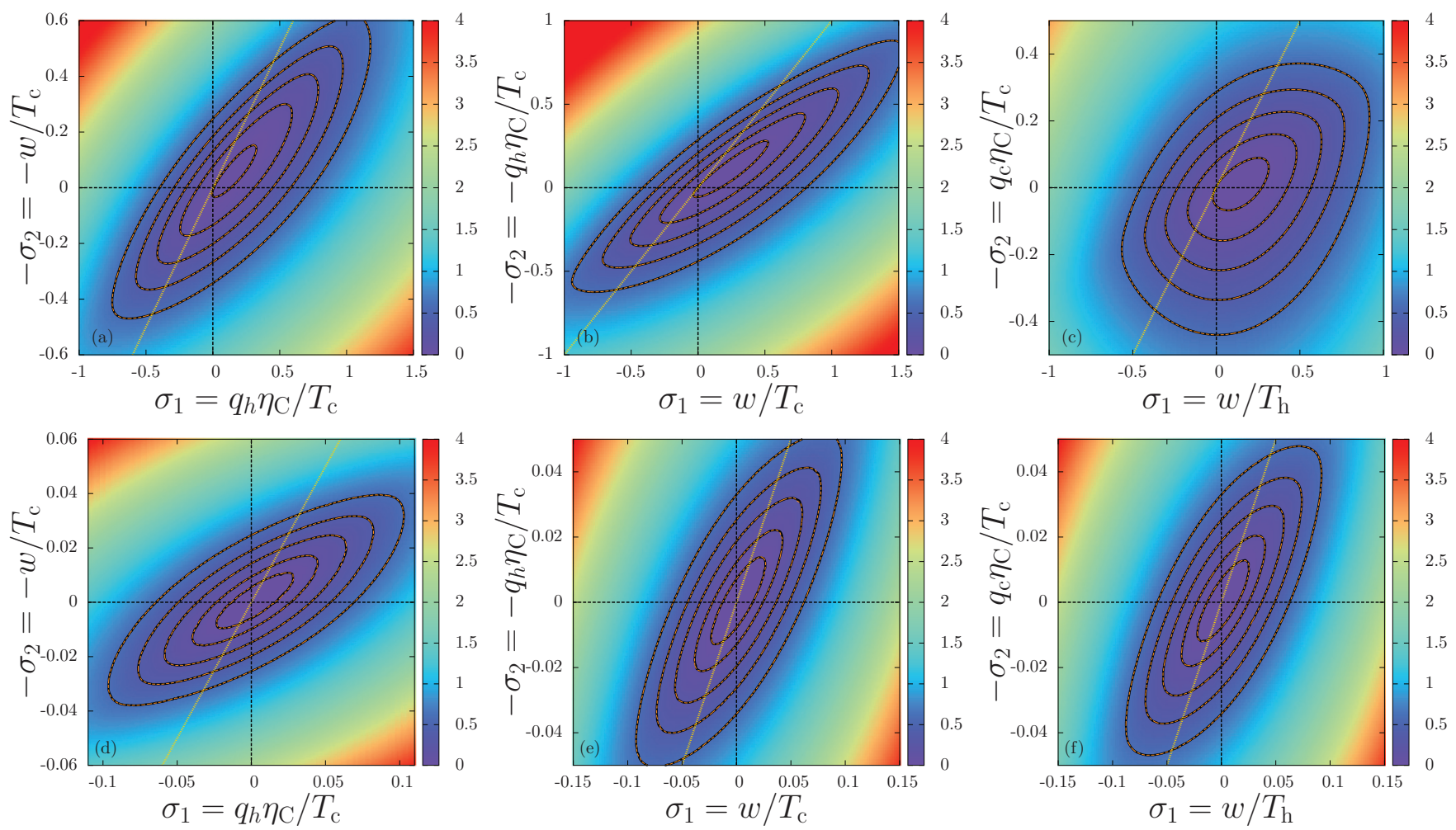

FIG. 6. (Color online) LDF of work and heat for the three operating modes of the machine: heat engine (a,d), heat pump (b,e), and refrigerator (c,f), far from (top row) or close to equilibrium (bottom row). The parameter values correspond to the black dots in Fig. 5. The solid black contour lines corresponding to $I\left(\sigma_{1}, \sigma_{2}\right)$ and the orange dashed lines corresponding to $I\left(-\sigma_{1},-\sigma_{2}\right)-\sigma_{1}-\sigma_{2}$ perfectly coincide, illustrating the fluctuation theorem for symmetric drivings under time-reversal. The yellow dotted line is the straight line of slope $\bar{\eta}_{\text {rev }}$ crossing the origin.

at the reversible efficiency are also very similar on Fig. 8 in far-from-equilibrium conditions, even though the position of the most probable efficiency is different. Finally, we remark that for all the parameter values and operating modes that

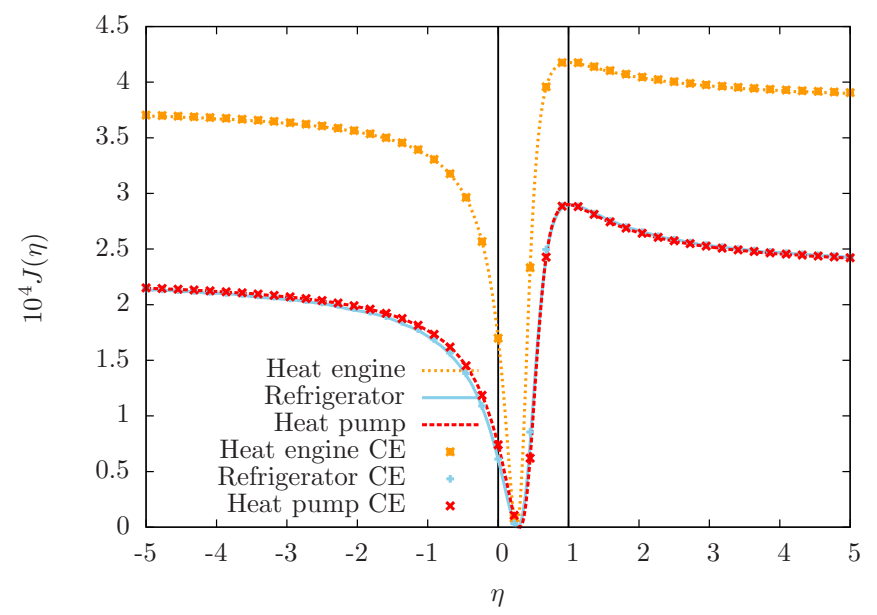

FIG. 7. (Color online) Efficiency LDF for the three main types of machines working close to equilibrium (CE). Lines are for $J(\eta)$ obtain from Eq. (26) and Table II and symbols come from the close-toequilibrium prediction of Eq. (31). The chosen parameters correspond to point I of Fig. 5 for the heat pump and the refrigerator and to point III of the same figure for the heat engine. The indicated type of machine corresponds to the average behavior. we considered, the general shape of the efficiency LDF is consistent with the one represented in Fig. 2.

In order to illustrate the general results of Sec. III C, we now consider the driving cycle depicted in Fig. 9, which is not symmetric under time reversal. We see in Fig. 10 that as anticipated, the reversible efficiency is not the least

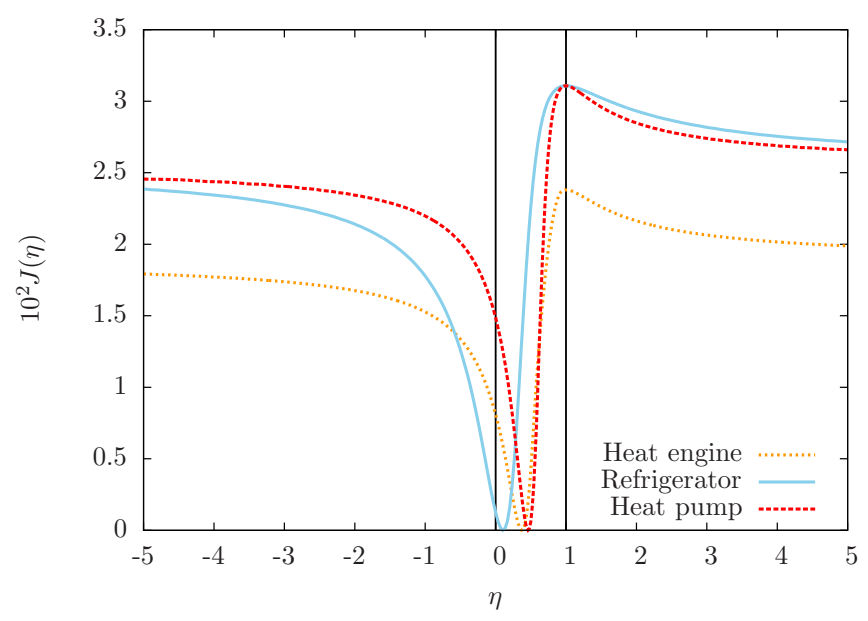

FIG. 8. (Color online) Efficiency LDF for the three main types of machines working far from equilibrium. The chosen parameters correspond to point II of Fig. 5 for the heat pump and the refrigerator and to point IV of the same figure for the heat engine. The indicated type of machine corresponds to the average behavior. 


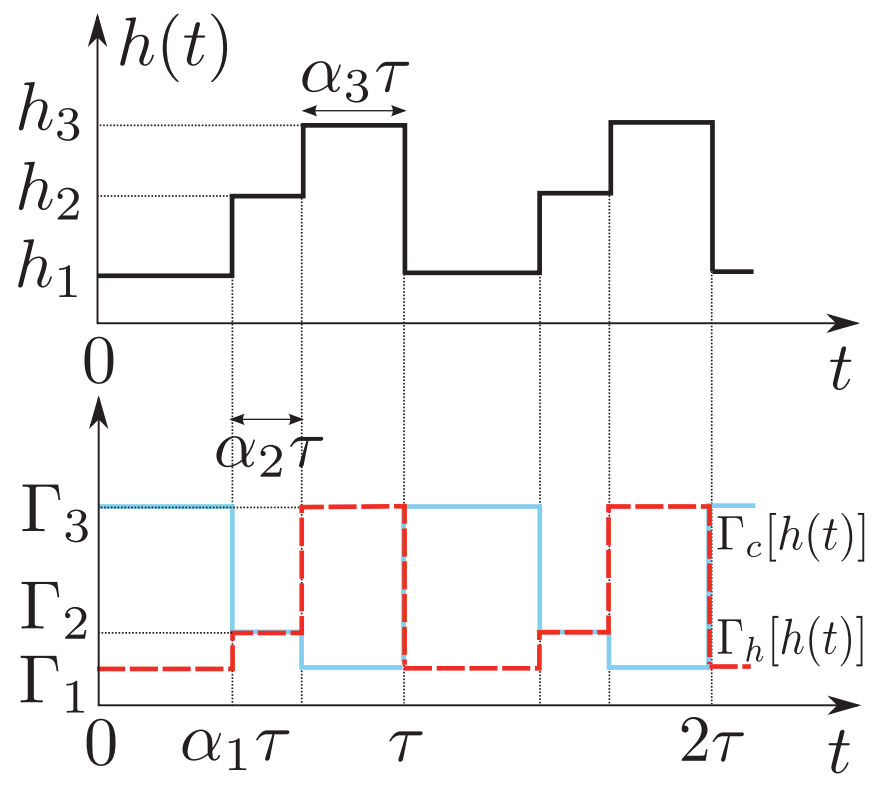

FIG. 9. (Color online) (Top) External driving following a piecewise constant protocol of period $\tau$. The driving takes three different values $h_{j}$ with $j=1,2,3$ during three time intervals $\alpha_{j} \tau$, with $\alpha_{1}+\alpha_{2}+\alpha_{3}=1$. (Bottom) Time evolution of $\Gamma_{v}(t)$ indicating the coupling with the cold reservoir $v=c$ for the blue solid line and the hot reservoir $v=h$ for the red dashed line. Note that the reverse driving cycle is defined by $\hat{h}(t)=h(\tau-t)$ and $\hat{\Gamma}_{v}(t)=\Gamma_{v}(\tau-t)$.

probable anymore but lies at the intersection of the forward and time-reversed driving curves. This is only clearly seen far from equilibrium since the effect of the time asymmetry of the driving vanishes as one approaches equilibrium as noted in the end of Sec. III E. A similar observation was recently made in Fig. 3 of Ref. [12].

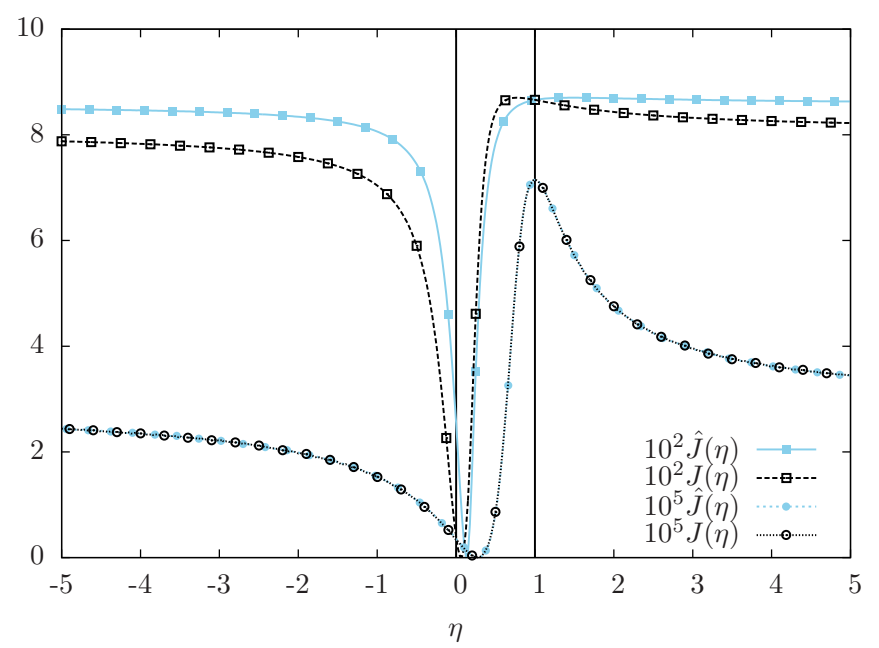

FIG. 10. (Color online) Efficiency LDF for a refrigerator (average behavior) working far from equilibrium (squares) or close to equilibrium (circles) with the asymmetric driving cycle under time reversal of Fig. 9. The empty (full) symbols are for the direct (reverse) driving. The kinetics is described by Fermi rates and the common parameters to all curves are $\alpha_{1}=0.6, \alpha_{2}=\alpha_{3}=0.2, \tau=1, \Gamma_{1}=$ $0.1, \Gamma_{2}=1, \Gamma_{3}=10$, and $\beta_{h}=1$. For far-from-equilibrium case, $h_{1}=0.5, h_{2}=1.5, h_{3}=2$, and $\beta_{c}=1.5$. For close-to-equilibrium case, $h_{1}=1.45, h_{2}=1.5, h_{3}=1.55$, and $\beta_{c}=1.05$.

\section{CONCLUSION}

We first summarize our results. Using the fluctuation theorem and assuming convexity of the currents LDF, we described the general properties of the LDF of efficiency fluctuations. Our conclusions hold for thermal and isothermal machines working arbitrarily far from equilibrium. We proved that the macroscopic efficiency defined as the ratio of average output power over average input power is the most probable efficiency. For general driving cycles, the reversible efficiency is special in that the efficiency LDF of a machine subjected to a forward driving cycle and that of the same machine driven by the time-reversal protocol, coincide at that point. For machines operating at steady state or subjected to time-symmetric driving cycles, the reversible efficiency is also the least likely efficiency as shown in Ref. [11]. In the close-to-equilibrium limit, the efficiency LDF is fully characterized by the response coefficients of the machine. Furthermore, in this regime, machines subjected to a driving cycle or its time-reversed version display the same efficiency LDF. We explicitly verified and illustrated our results by considering a two-level system machine subjected to piecewise constant driving protocols. We finally also proposed a very efficient method to calculate the efficiency LDF directly from the cumulant-generating function for the input and output currents.

Nowadays, stochastic quantities such as heat and work have been measured in various small systems [22-35] (e.g., biomolecules, systems of colloidal particles, polymers, quantum dots, single electron box). Hence their ratio, the stochastic efficiency, should be easily accessible experimentally. The statistical properties of the efficiency provide a much more accurate characterization of the performance of small machines than the macroscopic efficiency. In view of the high interest in recent years for the study of finite-time thermodynamics at small scales [7,35-44], we expect that the study of efficiency fluctuations will become a paradigm in this field. Finally, let us emphasize that the predictions of our theory for efficiency fluctuations provide a way to verify the implications of the fluctuation theorem, which can be seen as the generalization of the second law for small systems.

\section{ACKNOWLEDGMENTS}

This work was supported by the National Research Fund, Luxembourg, under Projects No. FNR/A11/02 and No. INTER/FWO/13/09. It also benefited from the COST action No. MP1209.

\section{APPENDIX: WORK AND HEAT CGF}

We derive here the work and heat CGF for the two-state model of Sec. IV with the driving of Fig. 4. In this model, transitions between system states require an instantaneous energy input or output, which corresponds to the heat exchanged with one of the reservoirs $v$. We use the label $v(t)$ to specify which reservoir caused the transition at time $t$. Energy conservation implies that the system energy change,

$$
\Delta E(t)=W(t)+Q_{c}(t)+Q_{h}(t),
$$


can be expressed as the sum of the work provided by the driving,

$$
W(t)=-\int_{0}^{t} d t^{\prime} \dot{h}\left(t^{\prime}\right) \theta\left(t^{\prime}\right)
$$

and the heat provided by the reservoirs,

$$
Q_{v}(t)=-\int_{0}^{t} d t^{\prime} h\left(t^{\prime}\right) \dot{\theta}\left(t^{\prime}\right) \delta_{v, v(t)}
$$

where $\delta$ is the Kronecker symbol.

For simplicity, we focus on the efficiency fluctuations of a refrigerator studying the statistics of work $W(t)$ and heat $Q_{c}(t)$. This implies no loss of generality: upon relabeling, we can also get the heat-engine or heat-pump efficiency fluctuations. The moment generating functions for work and heat at time $t$ conditioned on the final state $\theta$ is defined by

$$
G_{\theta}\left(\gamma_{1}, \gamma_{2}, t\right)=\left\langle e^{\gamma_{1} W(t)+\gamma_{2} Q_{c}(t)} \delta_{\theta, \theta(t)}\right\rangle .
$$

The one without conditioning is given by $G\left(\gamma_{1}, \gamma_{2}, t\right)=$ $\sum_{\theta} G_{\theta}\left(\gamma_{1}, \gamma_{2}, t\right)$. The evolution of Eq. (A4) is ruled by the master equation

$$
\partial_{t} G_{\theta}\left(\gamma_{1}, \gamma_{2}, t\right)=\sum_{\theta^{\prime}= \pm 1} L_{\theta, \theta^{\prime}}^{\left(\gamma_{1}, \gamma_{2}\right)}[h(t)] G_{\theta^{\prime}}\left(\gamma_{1}, \gamma_{2}, t\right)
$$

where $\boldsymbol{L}^{\left(\gamma_{1}, \gamma_{2}\right)}$ is a 2 by 2 matrix dependent of $h$ with elements

$$
L_{\theta, \theta^{\prime}}^{\left(\gamma_{1}, \gamma_{2}\right)}(h)=-\sum_{\nu=h, c} \theta \theta^{\prime} \omega_{\nu}(h) e^{-\beta^{\nu} \theta^{\prime} h+\gamma_{2} h\left(\theta^{\prime}-\theta\right) \delta_{\nu, l}}-\dot{h} \gamma_{1} \theta \delta_{\theta, \theta^{\prime}}
$$

This so-called "dressed" generator of the evolution is equal to the master equation generator for the probability of the system states when $\gamma_{1}$ and $\gamma_{2}$ vanish. The asymptotic CGF of work and heat is related to the highest eigenvalue $\rho\left(\gamma_{1}, \gamma_{2}\right)$ of the propagator over one period of Eq. (A5), written

$$
\boldsymbol{Q}=\overrightarrow{\exp } \int_{0}^{\tau} \boldsymbol{L}^{\left(\gamma_{1}, \gamma_{2}\right)}[h(t)] d t
$$

where $\overrightarrow{\exp }$ stands for the time-ordered exponential. To see this, we write $g_{\theta}\left(\gamma_{1}, \gamma_{2}\right)$ the right eigenvector of $\boldsymbol{Q}$ associated to $\rho\left(\gamma_{1}, \gamma_{2}\right)$ and $g\left(\gamma_{1}, \gamma_{2}\right)=\sum_{\theta} g_{\theta}\left(\gamma_{1}, \gamma_{2}\right)$ the sum of its components. Then, we have after $n$ periods

$$
\begin{aligned}
G\left(\gamma_{1}, \gamma_{2}, n \tau\right) & =\sum_{\theta, \theta^{\prime}}\left(\boldsymbol{Q}^{n}\right)_{\theta, \theta^{\prime}} g_{\theta^{\prime}}\left(\gamma_{1}, \gamma_{2}\right), \\
& =\rho\left(\gamma_{1}, \gamma_{2}\right)^{n} g\left(\gamma_{1}, \gamma_{2}\right),
\end{aligned}
$$

leading to the asymptotic CGF of work and heat (per period) coming from the cold reservoir,

$$
\varphi_{c}\left(\gamma_{1}, \gamma_{2}\right)=\lim _{n \rightarrow \infty} \frac{1}{n} \ln G\left(\gamma_{1}, \gamma_{2}, n \tau\right)=\ln \rho\left(\gamma_{1}, \gamma_{2}\right) .
$$

In other words, we have to compute the matrix $Q$ and look for its largest eigenvalue. This propagator follows from the product of four propagators for Eq. (A5): the propagator between time 0 and $\alpha^{-} \tau$ with the driving being $h^{-}$, the propagator over a unique time step during which occurs the transition from $h^{-}$to $h^{+}$- only the second term in the right hand side of Eq. (A6) matters for this propagation-the propagator between time $\alpha^{-} \tau$ and $\tau$ with the driving being $h^{+}$ and, finally, the propagation over the time step of the transition from $h^{+}$to $h^{-}$. These calculations have been described in more detail in Ref. [20] in the case of a modulated two-level system in contact with a unique heat reservoir, but the calculations here are essentially the same. The final result for the CGF is

$$
\varphi_{c}\left(\gamma_{1}, \gamma_{2}\right)=\ln \frac{\operatorname{tr} \boldsymbol{Q}+\sqrt{[\operatorname{tr} \boldsymbol{Q}]^{2}-4 \operatorname{det} \boldsymbol{Q}}}{2}
$$

which is a function of the determinant $\operatorname{det} \boldsymbol{Q}=z^{+} z^{-}$with $z^{ \pm}=\exp \left(-\tau \alpha^{ \pm} k^{ \pm}\right)$and $k^{ \pm}=2 \sum_{\theta, v} k_{v}\left(h^{ \pm}, \theta\right)$, and of the trace

$$
\begin{aligned}
\operatorname{tr} Q= & \sqrt{\frac{z^{+} z^{-}}{Z^{+} Z^{-}}}\left[1+Z^{+} Z^{-}+\left(1-Z^{+}\right)\left(1-Z^{-}\right)\right. \\
& \left.\times \frac{2 \mathcal{C}-K^{+} K^{-}}{2 K^{+} K^{-}}\right]
\end{aligned}
$$

with

$$
\begin{aligned}
& \mathcal{C}=\sum_{\substack{\mu, \nu=h, c \\
\epsilon= \pm}} \epsilon \omega_{\mu}^{-} \omega_{\nu}^{+} \cosh \left(\beta_{\epsilon}^{\mu \nu} h_{0}-\beta_{-\epsilon}^{\mu \nu} a\right) \\
& +\sum_{\mu, \nu=h, c} 2 \omega_{\mu}^{-} \omega_{\nu}^{+} \cosh \left[\left(\beta_{+}^{\mu \nu}-2 \gamma_{2}\left(\delta_{c, \mu}+\delta_{c, v}\right)+4 \gamma_{1}\right) a\right. \\
& \left.-\left(\beta_{-}^{\mu \nu}-2 \gamma_{2} \varepsilon_{\mu \nu}\right) h_{0}\right] .
\end{aligned}
$$

In these expressions, we have defined $\beta_{ \pm}^{\mu \nu}=\beta_{\mu} \pm \beta_{v}$ for the sum and difference of inverse temperatures, used the short notation $\omega_{v}\left(h^{ \pm}\right)=\omega_{v}^{ \pm}$, and introduced the LeviCivita tensor for the heat reservoirs $\varepsilon_{c c}=\varepsilon_{h h}=0, \varepsilon_{c h}=1$, and $\varepsilon_{h c}=-1$. We have also defined $Z^{ \pm}=\exp \left(-\tau \alpha^{ \pm} K^{ \pm}\right)$ and

$$
\begin{aligned}
K^{ \pm}= & \left\{4 \sum _ { \mu , \nu } \omega _ { \mu } ^ { \pm } \omega _ { v } ^ { \pm } \left[\cosh h^{ \pm}\left(\beta_{-}^{\mu \nu}-2 \gamma_{2} \varepsilon_{\mu \nu}\right)\right.\right. \\
& \left.\left.-\cosh h^{ \pm} \beta_{-}^{\mu \nu}\right]+\left(k^{ \pm}\right)^{2}\right\}^{1 / 2}
\end{aligned}
$$

We observe in Eqs. (A11)-(A14) that we have $\varphi_{c}\left(\gamma_{1}, 0\right) \sim$ $\pm 4 a \gamma_{1}$ in the limit $\gamma_{1} \rightarrow \pm \infty$ : the maximum slope of the CGF is $\pm 4 a$, which is consistent with the extremal work values for this model. The heat exchanges are in principle unbounded; this corresponds, in the large $\left|\gamma_{2}\right|$ limit, to the fact that the CGF increases exponentially (no bounds on the slopes). As announced, the CGF of work and heat coming from the hot 
TABLE II. Connection between the CGF $\phi$ of the EPs per unit time and the CGF of the work and heat per period.

\begin{tabular}{cccc}
\hline \hline & Heat engine & Heat pump & Refrigerator \\
\hline$\phi\left(\gamma_{1}, \gamma_{2}\right)$ & $\varphi_{h}\left(\gamma_{2} / T_{c}, \gamma_{1} \eta_{C} / T_{c}\right) / \tau$ & $\varphi_{h}\left(\gamma_{1} / T_{c}, \gamma_{2} \eta_{C} / T_{c}\right) / \tau$ & $\varphi_{c}\left(\gamma_{1} / T_{h},-\gamma_{2} \eta_{C} / T_{c}\right) / \tau$ \\
\hline \hline
\end{tabular}

reservoir is defined by

$$
\varphi_{h}\left(\gamma_{1}, \gamma_{2}\right)=\lim _{n \rightarrow \infty} \frac{1}{n} \ln \left\langle e^{\gamma_{1} W(t)+\gamma_{2} Q_{h}(t)}\right\rangle,
$$

and is obtained exchanging the labels $c$ and $h$ in Eqs. (A11)(A14). The CGF $\varphi_{c}\left(\gamma_{1}, \gamma_{2}\right)$ and $\varphi_{h}\left(\gamma_{1}, \gamma_{2}\right)$ provide all the required information to study the efficiency fluctuations of the three types of thermal machines as shown in Table II.

Evaluating the derivative of the CGF of work and heat at the origin, one obtains the average work per period,

$$
\langle w\rangle=\frac{8 a \mathcal{Z}}{k^{-} k^{+}} \sum_{\mu \nu} \omega_{\mu}^{-} \omega_{v}^{+} \sinh \left(\beta_{+}^{\mu v} a-\beta_{-}^{\mu v} h_{0}\right),
$$

and the average heat per period coming from the cold reservoir,

$$
\begin{aligned}
\left\langle q_{c}\right\rangle= & 4 \sum_{\epsilon= \pm}\left[\frac{\mathcal{Z}}{k^{\epsilon} k^{\epsilon}}-\frac{\tau \alpha^{\epsilon}}{k^{\epsilon}}\right] \omega_{c}^{\epsilon} \omega_{h}^{\epsilon} h^{\epsilon} \sinh \beta_{-}^{c h} h^{\epsilon} \\
& -\frac{4 \mathcal{Z}}{k^{-} k^{+}}\left[\sum_{\epsilon= \pm} \epsilon \omega_{c}^{\epsilon} \omega_{h}^{-\epsilon} h^{\epsilon} \sinh \left(\beta_{+}^{c h} a+\epsilon \beta_{-}^{c h} h_{0}\right)\right. \\
& \left.+2 a \omega_{c}^{-} \omega_{c}^{+} \sinh 2 \beta_{c} a\right]
\end{aligned}
$$

where we have defined $\mathcal{Z}=\left(1-z^{-}\right)\left(1-z^{+}\right) /\left(1-z^{-} z^{+}\right)$. As for the generating function, the average heat from the hot reservoir $\left\langle q_{h}\right\rangle$ is obtained by interchanging all the labels $c$ with $h$.
[1] U. Seifert, Phys. Rev. Lett. 95, 040602 (2005).

[2] M. Esposito and C. Van den Broeck, Phys. Rev. Lett. 104, 090601 (2010).

[3] G. Verley, R. Chétrite, and D. Lacoste, Phys. Rev. Lett. 108, 120601 (2012).

[4] K. Sekimoto, Stochastic Energetics, Lect. Notes Phys. 799 (Springer, Berlin Heidelberg, 2010).

[5] N. A. Sinitsyn, J. Phys. A: Math. Theoret. 44, 405001 (2011).

[6] M. Campisi, J. Phys. A: Math. Theoret. 47, 245001 (2014).

[7] U. Seifert, Rep. Prog. Phys. 75, 126001 (2012).

[8] C. Van den Broeck and M. Esposito, Phys. A 418, 6 (2014).

[9] M. Campisi, P. Hänggi, and P. Talkner, Rev. Mod. Phys. 83, 771 (2011).

[10] M. Esposito, U. Harbola, and S. Mukamel, Rev. Mod. Phys. 81, 1665 (2009).

[11] G. Verley, M. Esposito, T. Willaert, and C. Van den Broeck, Nat. Commun. 5, 4721 (2014).

[12] T. R. Gingrich, G. M. Rotskoff, S. Vaikuntanathan, and P. L. Geissler, New J. Phys. 16, 102003 (2014).

[13] A. Bejan, Advanced Engineering Thermodynamics (Wiley, New York, 2006).

[14] R. García-García, D. Domínguez, V. Lecomte, and A. B. Kolton, Phys. Rev. E 82, 030104 (2010).

[15] R. García-García, V. Lecomte, A. B. Kolton, and D. Domínguez, J. Stat. Mech. (2012) P02009.

[16] J. L. Lebowitz and H. Spohn, J. Stat. Phys. 95, 333 (1999).

[17] P. Gaspard, J. Chem. Phys. 120, 8898 (2004).

[18] M. Esposito, U. Harbola, and S. Mukamel, Phys. Rev. E 76, 031132 (2007).

[19] H. Touchette, Phys. Rep. 478, 1 (2009).

[20] G. Verley, C. Van den Broeck, and M. Esposito, Phys. Rev. E 88, 032137 (2013).

[21] G. Verley, C. Van den Broeck, and M. Esposito, New J. Phys. 16, 095001 (2014).
[22] O.-P. Saira, Y. Yoon, T. Tanttu, M. Möttönen, D. V. Averin, and J. P. Pekola, Phys. Rev. Lett. 109, 180601 (2012).

[23] J. R. Moffitt, Y. R. Chemla, K. Aathavan, S. Grimes, D. L. Anderson, and C. Bustamante, Nature 457, 446 (2009).

[24] R. Yasuda, H. Noji, M. Yoshida, K. Kinosita, and H. Itoh, Nature 410, 898 (2001).

[25] S. Toyabe, T. Sagawa, M. Ueda, E. Muneyuki, and M. Sano, Nat. Phys. 6, 988 (2010).

[26] A. Bérut, A. Arakelyan, A. Petrosyan, S. Ciliberto, R. Dillenschneider, and E. Lutz, Nature 483, 187 (2012).

[27] A. Alemany, A. Mossa, I. Junier, and F. Ritort, Nat. Phys. 8, 688 (2012).

[28] D. Collin, F. Ritort, C. Jarzynski, S. B. Smith, I. J. Tinoco, and C. Bustamante, Nature 437, 231 (2005).

[29] J. V. Koski, T. Sagawa, O.-P. Saira, Y. Yoon, A. Kutvonen, P. Solinas, M. Möttönen, T. Ala-Nissila, and J. P. Pekola, Nat. Phys. 9, 644 (2013).

[30] B. Küng, C. Rössler, M. Beck, M. Marthaler, D. S. Golubev, Y. Utsumi, T. Ihn, and K. Ensslin, Phys. Rev. X 2, 011001 (2012).

[31] S. Ciliberto, A. Imparato, A. Naert, and M. Tanase, Phys. Rev. Lett. 110, 180601 (2013).

[32] C. Bustamante, J. Liphardt, and F. Ritort, Phys. Today 58(7), 43 (2005).

[33] J. Matthews, F. Battista, D. Sanchez, P. Samuelsson, and H. Linke, Phys. Rev. B 90, 165428 (2014).

[34] S. Tusch, A. Kundu, G. Verley, T. Blondel, V. Miralles, D. Démoulin, D. Lacoste, and J. Baudry, Phys. Rev. Lett. 112, 180604 (2014).

[35] V. Blickle and C. Bechinger, Nat. Phys. 8, 143 (2012).

[36] M. Esposito, N. Kumar, K. Lindenberg, and C. Van den Broeck, Phys. Rev. E 85, 031117 (2012).

[37] M. Esposito, R. Kawai, K. Lindenberg, and C. Van den Broeck, Phys. Rev. E 81, 041106 (2010). 
[38] M. Esposito, K. Lindenberg, and C. Van den Broeck, Phys. Rev. Lett. 102, 130602 (2009).

[39] U. Seifert, Phys. Rev. Lett. 106, 020601 (2011).

[40] T. Schmiedl and U. Seifert, Europhys. Lett. 81, 20003 (2008).

[41] Z. C. Tu, Phys. Rev. E 89, 052148 (2014).
[42] N. Golubeva and A. Imparato, Phys. Rev. Lett. 109, 190602 (2012).

[43] G. Benenti, G. Casati, T. Prosen, and K. Saito (2013), arXiv:1311.4430.

[44] B. Cleuren, B. Rutten, and C. Van den Broeck, Phys. Rev. Lett. 108, 120603 (2012). 